\title{
Pulmonary vein anatomy variants as a biomarker of atrial fibrillation - CT angiography evaluation
}

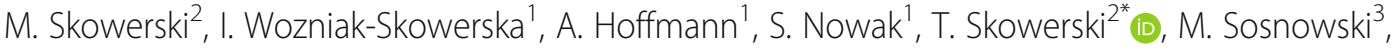 \\ A. M. Wnuk-Wojnar ${ }^{1}$ and K. Mizia-Stec ${ }^{1}$
}

\begin{abstract}
Background: It has been suggested that changes in pulmonary veins (PV) and left atrium (LA) anatomy may have an influence on initiating atrial fibrillation (AF) and the effectiveness of pulmonary vein isolation (PVI) in patients (pts) with atrial fibrillation.

The aim of the study was to assess anatomy abnormalities of the PV and LA in the patients with the history of AF and compare it with the control group(CG).

Methods: The multi-slice tomography (MSCT) scans were performed in 224 AF pts. before PVI (129 males, mean age $59 \pm 9$ yrs). The CG consisted of 40 pts. without AF (26 males, age $45 \pm 9$ yrs). LA and PV anatomy were evaluated. Diameters of PV ostia were measured in two directions: anterior-posterior (AP) and superior-inferior (SI) automatically using Vitrea 4.0.

Results: Pulmonary veins anatomy variants were observed more frequently in the atrial fibrillation group - 83 pts. (37\%) vs 6 pts. (15\%) in CG; 9\% (21 pts) left common ostia (CO), 2\% (5 pts) right CO, 19\% (42 pts) additional right PV (APV), (1.8\%) 4 pts. APV left, 8\% right early branching (EB) and 3.5\% left EB. The LA diameter differed significantly in AF vs CG group $(41.2 \pm 6 \mathrm{~mm}$ vs $35 \pm 4.2 \mathrm{~mm}, p<0.0001)$ respectively.

Conclusions: The anomalies of pulmonary vein anatomy occurred more often in pts. with AF. They can be defined as an image biomarkers of atrial fibrillation. Right additional (middle) pulmonary vein was the most important anomaly detected in AF patients as well as enlargered diameters of the LA and PV ostia.
\end{abstract}

Keywords: Atrial fibrillation, Pulmonary vein isolation, CT angiography

\section{Background}

Atrial fibrillation (AF) is a frequently occurring arrhythmia that impairs the life functioning, is associated with the increasing medical supervision, the risk of other diseases and pharmacological therapy complications. Pulmonary vein isolation (PVI) over the last decade has become the most demanded method for AF treatment. Even with experience in ablation, knowledge of left atrial anatomy and pulmonary vein anatomy is necessary [1]. A detailed visualization of the left atrium (LA) and pulmonary vein (PV) anatomy can be obtained by several different imaging methods including

\footnotetext{
* Correspondence: tskowerski@gmail.com

${ }^{2}$ Department of Cardiology, School of Health Sciences, Medical University of Silesia, Katowice, Poland

Full list of author information is available at the end of the article
}

transesophageal or intracardiac echocardiography, rotational angiography, multislice computed tomography (MSCT) or three-dimensional gadolin-enhanced magnetic resonance. The MSCT is one of the most common and objective methods [2-5]. Anatomic varations of left atrium such as common ostia, additional pulmonary vein or early branching are common and previously described.

Some authors suggest that those anomalies may play a significant role in the pathophysiology of atrial fibrillation and even increase its prevalence $[4,6]$. The safety and effectiveness of PVI in patients with AF is still under intensive clinical investigation [5, 7]. Therefore, the purpose of this study was to evaluate the occurrence of anatomy anomalies of $\mathrm{PV}$ and the left atrium using

(c) The Author(s). 2018 Open Access This article is distributed under the terms of the Creative Commons Attribution 4.0 International License (http://creativecommons.org/licenses/by/4.0/), which permits unrestricted use, distribution, and 
MSCT scans performed in patients with AF and compare the results with no history of AF (control group).

\section{Methods}

\section{Study population}

A total of 271 pts. are analyzed in this paper. The study population (AF group) consisted of 224 patients (129 males, mean age $59 \pm 9$ yrs., range $22-74$ yrs) with the history of paroxysmal and/or persistent symptomatic non-valvular AF referred to our hospital for qualification for PVI. The study was approved by the ethics committee and conformed to the Declaration of Helsinki. An informed written consent was obtained from every patient enrolled in the study. All the patients from the study group had well documented episodes of AF using Holter ECG and/or surface ECG. MSCT images were performed for LA and PV visualization. Exclusion criteria involved: history of congenital heart diseases, left ventricular systolic dysfunction (LV EF $<35 \%$ ), hemodynamically significant valvular heart diseases. The subjects who underwent PVI were also excluded from the study.

The control group (CG) consisted of 40 pts. (26 males, mean age $45 \pm 9$ yrs) with no history of AF. MSCT scans were performed due to the symptoms of suspected pathologies in a coronary artery disease and/or aortic disease, which were finally excluded. The clinical characteristics of the study population and the control group are presented in Table 1.

Seven patients were excluded from the study due to rare anomalies that are described in the results.

\section{MSCT of PVs}

Multislice computed tomography (MSCT) scans were performed in all patients with a 64-slice Toshiba Multislice Aquilion System (Toshiba Medical System, Japan). Retrospective electrocardiographic gating was performed to minimize cardiac motion artifacts.

Non-ionic contrast material (Ultravist 370, Schering AG) was injected in the antebrachial vein $(120 \mathrm{ml}$ in three phases, phase one- $70 \mathrm{ml}$, flow $5.0 \mathrm{ml} / \mathrm{s}-100 \%$ contrast, phase two- $30 \mathrm{ml}$ the same flow- $60 \%$ contrast and

Table 1 Baseline characteristics of AF patients and CG

\begin{tabular}{lll}
\hline & AF $(n=224)$ & CG $(n=40)$ \\
\hline Age (years) & $59 \pm 9$ & $47 \pm 8$ \\
Gender (M/F) & $129 / 95$ & $16 / 15$ \\
Hypertension & $140(63 \%)$ & $17(53 \%)$ \\
BMI > 25 & $152(68 \%)$ & $14(45 \%)$ \\
DM & $23(10 \%)$ & 0 \\
Ml & $15(7 \%)$ & 0 \\
Stroke & $11(5 \%)$ & 0 \\
LVEF ECHO (\%) & $54 \pm 9$ & $60 \pm 5$
\end{tabular}

$M I$, myocardial infarction, $D M$ diabetes mellitus, $B M I$ body mas index
$40 \%$ saline, phase three- $20 \mathrm{ml}, 4.0 \mathrm{ml} / \mathrm{s}$ flow- $100 \%$ saline, by means of a dual-head power injector-Injector CT2, Medtron, Germany).

Data sets were subsequently analyzed on Vitrea post-processing workstation (Vital Images) using 2-D and $3-\mathrm{D}$ viewing modes. Electrocardiographically gated datasets were reconstructed automatically at different time of the R-R cycle length to approximate end-diastole phase of the cardiac cycle. Additional reconstruction windows were constructed after the examination of datasets if motion artifacts were present. Scans were analyzed by consensus of two observers. Images were evaluated using $0.5 \mathrm{~mm}$ thin-slab maximum intensity projections (MIP) and curved multiplanar reconstructions (cMPR).

LA diameters were measured with the maximal anterior-posterior distance in the oblique-sagittal view. The PV anatomy was assessed - the number of PV, common ostia and the branching pattern. The diameters of PV ostia were measured in two directions (anterior-posterior (AP) and superior-inferior (SI)). We defined the anomalies as follows in accordance with previous publications $[3,6,8]$ :

- Additional pulmonary vein (APV) was defined as an extra PV up to pattern of 4 PVs.

- Common ostia (CO) was defined when the distance between the virtual border of the LA and the bifurcation of both PVs was $0.5 \mathrm{~cm}$ or less.

- Early branching (EB) was specified as the bifurcation of the PV within $1 \mathrm{~cm}$ of origin from the LA.

- Venous ostium index (VOI) determined the ovality shape of ostia and was calculated by dividing MSCT measurements in the AP and the SI directions. When the ratio approaches 1.0 the ostium is more rounded, when it deviates from 1.0 the shape is more oval.

Endoluminal views were routinely rendered and allowed a precise visualisation of the pulmonary vein ostia, pulmonary vein orientation, distance to the first branch, geometry of pulmonary vein branches and common ostia.

\section{Statistical analysis}

The baseline clinical parameters and the results of parametric data were compared using the two-sample $t$-tests for normally distributed continuous variables between AF and CG group (Student's t-test or Wilcoxon matched paired test). The Chi-square test was used to analyze nonparametric data. All of the text and table results are expressed as means \pm standard deviation $(\mathrm{SD})$ from the mean or a number (percentage). Statistical evaluation was performed using the software Statistica ver. 8.0 Stat 
Soft Pl. $P$ value $<0.05$ was considered statistically significant.

\section{Results}

\section{LA dimension}

The mean value (measured in CT) of the LA size was significantly larger in AF group vs CG group (41.2 \pm $6 \mathrm{~mm}$ vs $35.4 \pm 4 \mathrm{~mm}, p<0.0001)$. Twenty six $(11.6 \%)$ of AF pts. had LA larger than $50 \mathrm{~mm}$, in comparison to CG, were no pts. had LA so enlarged.

\section{Anatomical anomalies}

All data are presented in Table 2. The typical anatomical pattern of the pulmonary vein described as two right and two left PVs was observed more often in CG than in AF group - 34 pts. (85\%) vs 150 pts. (67\%) pts.; $p<0.05$.

PV anatomy anomalies - additional pulmonary veins, common ostia or early branching were found more frequently in the AF group - 83 pts. (37\%) as compared to the CG -6 pts. (15\%); $\mathrm{p}<0.05$.

Forty two (18.7\%) pts. from AF group had additional right PV (APV), contrary to the CG where no APVs were observed. The mean diameter of APV's was significantly smaller than the mean diameter of main veins (AP $7.2 \pm 1.6 \mathrm{~mm}$, SI $7.3 \pm 1.8 \mathrm{~mm}$ vs AP $14.7 \pm 3.0 \mathrm{~mm}$, SI $17.4 \pm 2.4 \mathrm{~mm}, p<0.0001)$.

We recognized two types of common ostia of PVs:

- a long trunk CO with more than $20 \mathrm{~mm}$ distance between the left atrium and the bifurcation (5 AF pts., 0 CG pts)

- a short trunk CO - less than $20 \mathrm{~mm}$ distance between the left atrium and the bifurcation (21 AF pts., 6 CG pts).

The mean size of common ostia in AF patients was significantly larger than the diameter of main veins (AP 16.7 $\pm 4.2 \mathrm{~mm}$, SI $24.5 \pm 5.5 \mathrm{~mm}, \mathrm{p}<0.0001)$. There was a trend towards left-sided single ostia especially in AF patients.

There was no difference in the occurrence of early branching (EB) in AF - 5\% (11 pts) as compared to CG $5 \%$ (2 pts).

Table 2 Results: comparison of anatomical anomalies in AF patients and CG pts.

\begin{tabular}{lllll}
\hline & & AF $(n=224)$ & CG $(n=40)$ & $p$ \\
\hline LA CT $(\mathrm{mm})$ & & $41.2 \pm 6$ & $35.4 \pm 4$ & $<0.0001$ \\
Additional PV & right & $42(18.7 \%)$ & 0 & $<0.05$ \\
& left & $4(1.8 \%)$ & 0 & 0.45 \\
Common ostium & right & $5(2 \%)$ & $2(6.4 \%)$ & 0.13 \\
& left & $21(9 \%)$ & $4(12,9 \%)$ & 0.5 \\
Other anomalies & & $6(2.7 \%)$ & 0 & \\
\hline
\end{tabular}

In the study group some other anomalies were discovered:

- two additional PVs (right sided) - 1 pt. (Fig. 1);

- two additional PVs on the left and right side -1 pt.;

- single left PV - 1 pt. (Fig. 2);

- and left common ostia with a right additional PV (middle PV) - in 2 pts. (Fig. 3);

- abnormality of PV localization: all four left PVs were close to each other on the backside of LA (Fig. 4).

\section{Pulmonary vein ostia}

The AP and SI diameters of the PV ostia were significantly larger in the atrial fibrillation group than in the control group - results are shown in Table 3 . In both groups the mean ostial diameter of the superior PV was larger than that of the inferior PV.

The venous ostium index (VOI) calculated for each PV proves the oval shape of most of them. Only the VOI of the right inferior pulmonary vein (RIPV) did differ between the groups $(p<0.005)$. Table 4 presents the VOI ratios.

\section{Incidental findings}

We also found rare anomalies in 7 pts. with AF - they were excluded from the analysis but we find it worth presenting. Partially anomalous pulmonary venous return was observed in 2 pts., atrial septal defect with anomalous insertion right upper $\mathrm{PV}$ into the right atrium - in 1 pt., and anomalous insertion of the middle meningeal vein into the right upper pulmonary vein - in $1 \mathrm{pt}$. One patient had right atrial diverticulum and $1 \mathrm{pt}$. left atrial diverticulum with a clot. No other clots were found in the LA. Right atrial myxoma localised in the interatrialis septum was diagnosed in 1 pt.

\section{Discussion}

Several researchers, cardiologists, radiologists and surgeons studied the anatomy of LA and PV because of a variety of endovascular and surgical techniques used for invasive therapy of patients with AF [2-7].

This study once again proves the existence of significant differences in anatomy of LA and PV in AF patients in comparison with healthy individuals $[2,3]$. This study presents a significantly larger group of pts. (224 pts) with AF than our previous publication [8] (82 pts) and other previously published papers - Bittner et al. [9] (166 pts. with AF.), Kubala et al. [7] 118 pts. with AF. Also the number of PV anomalies is greater than in other studies. [7-10]. In reference to Chen (710 pts. with AF, 710 pts. CG) et al. [11] our study is consistent with the dimensions of PVs - larger PVs in AF group, in contrast to our findings in Chen's study the occurrence of $\mathrm{PV}$ variants did not differ between the groups. On the 

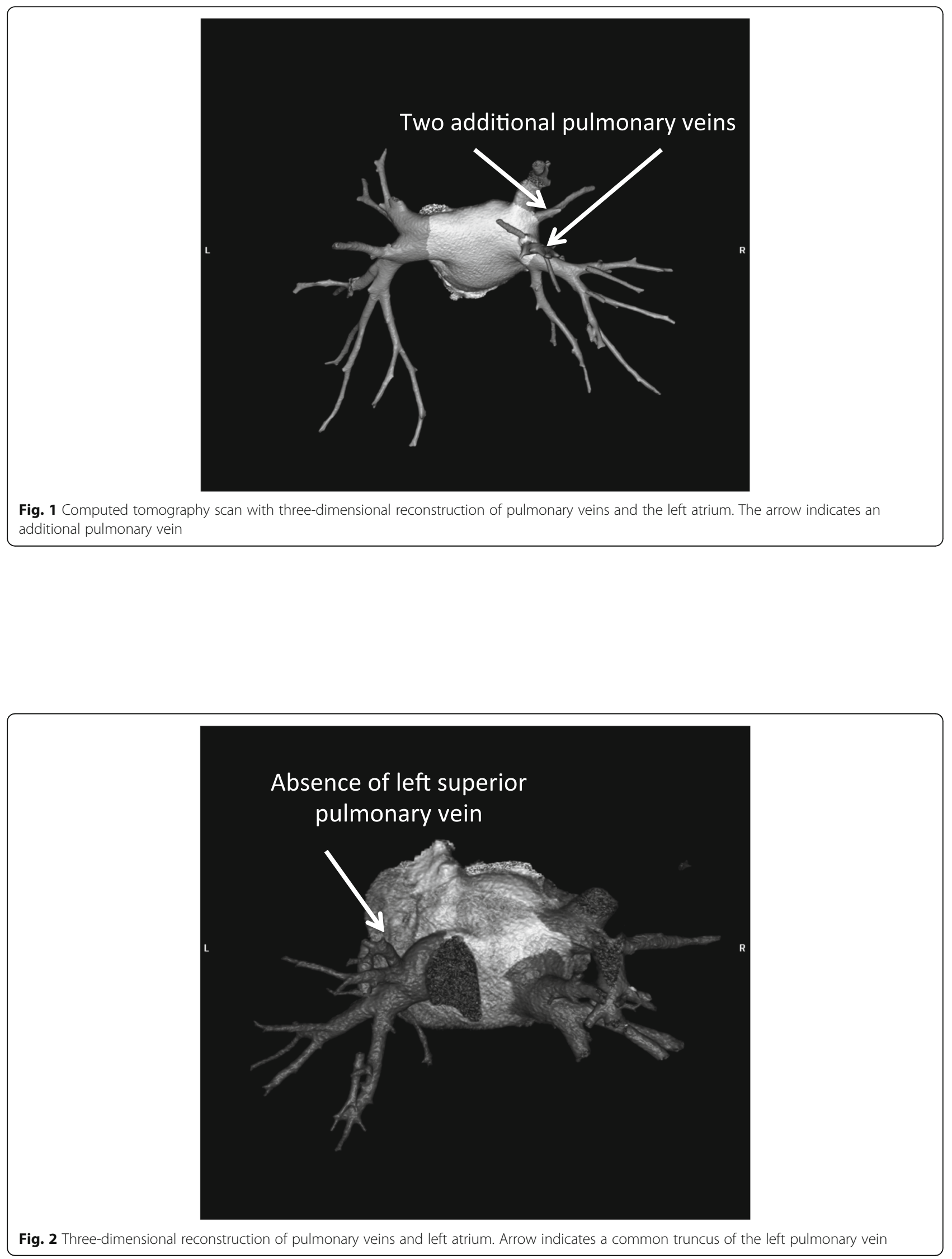


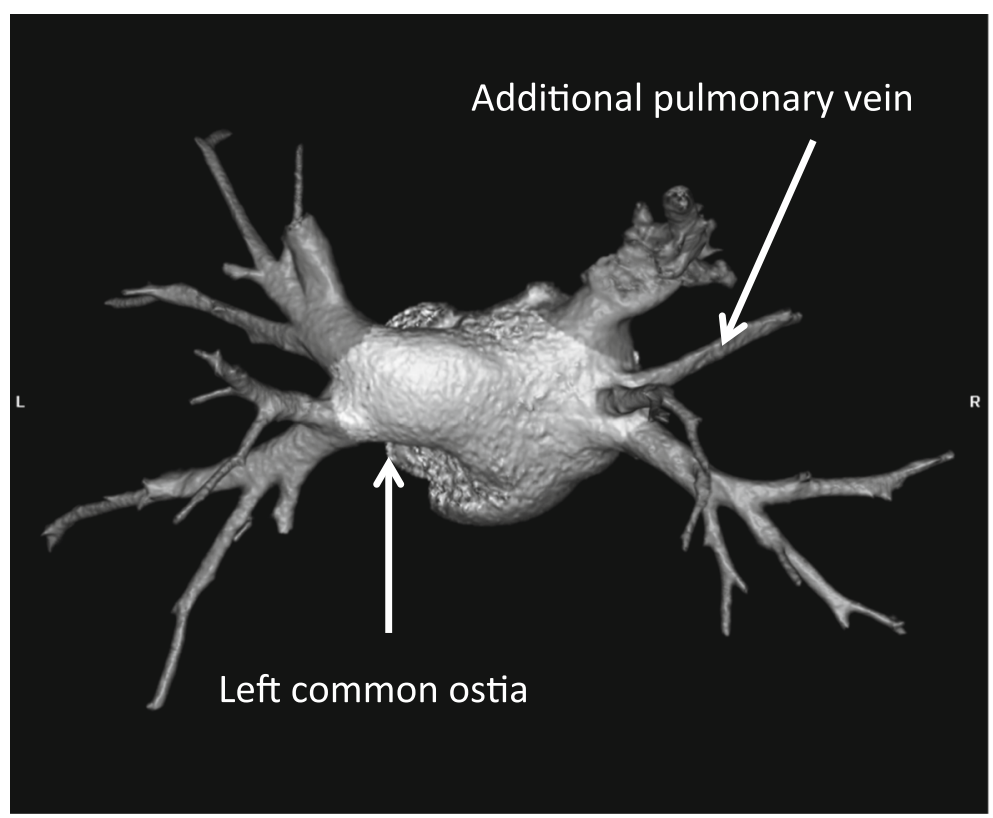

Fig. 3 Left common ostia with right additional PV (middle PV)

other hand one of the most extensive studies describing PV anatomy by Tekbas et al. shows the prevalence of PV abnormalities only in $26(3,3 \%)$ out of 783 patients without atrial fibrillation [12].

The VOI ratio defining the shape of the pulmonary vein ostium is useful information for the operator - some research suggest it may define the method of ablatoin (cry ablation vs radio-frequency), predict the outcome and the complications (such as PV occlusion) [13].

Prior studies have proven that MSCT and cardiac magnetic resonance (CMR) are appropriate, non-invasive, widely available tools for describing LA anatomy and PV

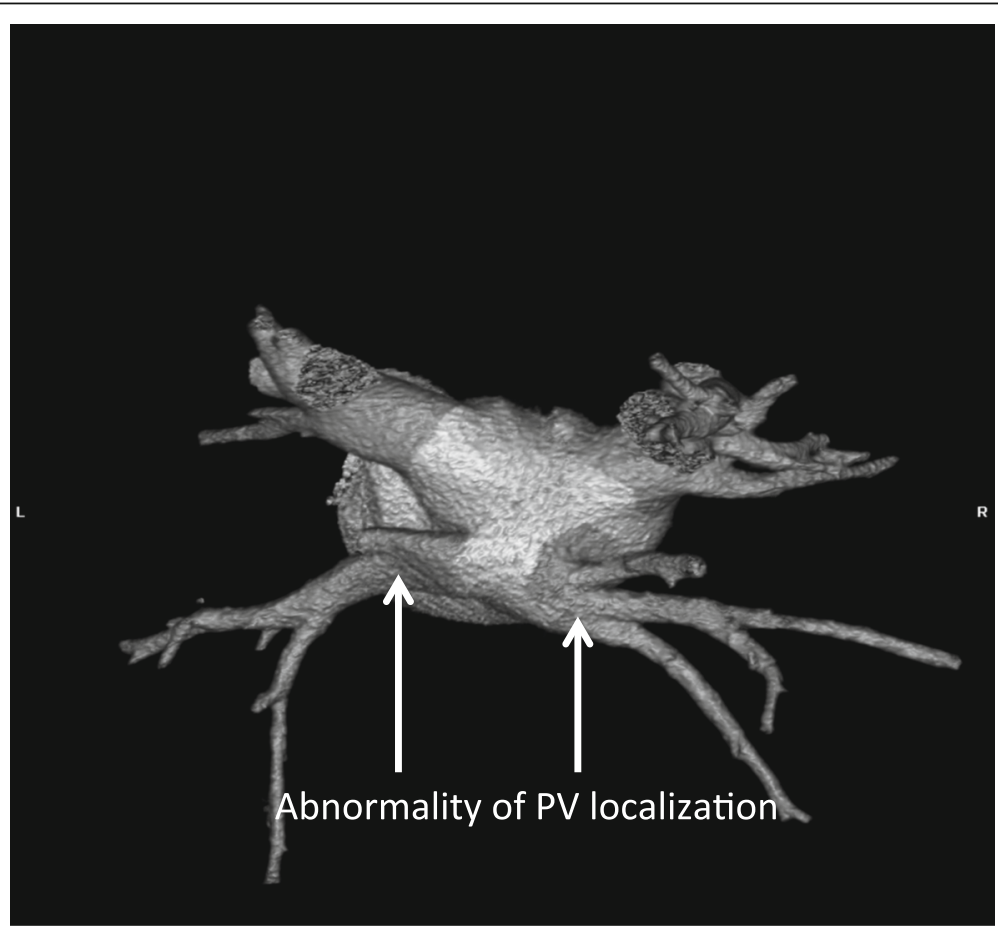

Fig. 4 Abnormality of PV localization: all (4) PVs left were close to each other on the posterior wall of LA 
Table 3 Comparison results of SI and AP diameters of typical pattern 4 PVs in AF patients and CG (mean $\pm S D$ )

\begin{tabular}{llll}
\hline Pulmonary veins & AF $(n=224)$ & CG $(n=40)$ & $p$ \\
\hline SI in mm & & & \\
RSPV & $18.5 \pm 2.0$ & $14.8 \pm 2.9$ & $<0.0001$ \\
RIPV & $16.8 \pm 1.9$ & $13.9 \pm 3.4$ & $<0.0001$ \\
LSPV & $18.2 \pm 2.6$ & $15.1 \pm 2.3$ & $<0.0001$ \\
LIPV & $16.1 \pm 3.1$ & $13.7 \pm 2.7$ & $<0.0001$ \\
AP in mm & & & \\
RSPV & $16.5 \pm 3.0$ & $13.7 \pm 2.9$ & $<0.0001$ \\
RIPV & $15.2 \pm 2.1$ & $13.6 \pm 3.4$ & $<0.0001$ \\
LSPV & $14.7 \pm 2.7$ & $12.0 \pm 2.5$ & $<0.0001$ \\
LIPV & $12.4 \pm 4.3$ & $10.3 \pm 3.7$ & $<0.001$ \\
\hline
\end{tabular}

$R S P V$ right superior pulmonary vein, RIPV right inferior pulmonary vein, $L S P V$ left superior pulmonary vein, LIPV left inferior pulmonary vein

$\mathrm{AF}(+)$ : right superior vs. right inferior PV $-\mathrm{SI} p<0.0001, \mathrm{AP} p<0.02$

left superior vs. left inferior PV - SI $p<0.0001, \mathrm{AP} p<0.0001$,

GC: right superior vs. right inferior PV - SI $p<0.005$, AP NS,

left superior vs left inferior PV $-\mathrm{SI} p<0.05, \mathrm{AP} p<0.05$

attachments in the patients qualified for the ablation procedure [2-16]. Moreover, CMR images allow for non-invasive therapy stratification using the atrial wall tissue characterization map for fibrosis assessment. It seems to be a good predictor for procedural outcome [2-10]. Although it has to be noticed that nowadays the left atrial model reconstruction with imaging by the electroanatomical mapping system is more often performed before the ablation procedure and CT/CMR imaging is not necessary to achieve good results and/or minimize complications $[17,18]$. Thus it doesn't change the significance of our findings.

Similarly to previous observations [2, 3, 8, 19-21] the data collected confirm larger diameters of LA, superior and inferior PVs in patients with AF. We want this study to underline the complexity of anatomical changes that may trigger AF.

It has been suggested that those anomalies may affect the results of pulmonary vein ablation [7, 8, 22-24]. Kubala et al. describes a superior clinical outcome in patients with typical 4 PV pattern in comparison with the patients with the left CO [7]. Sohns et al. analyzed 138 pts. and proved the impact of PV anatomy on AF recurrence after PVI [24]. Mulder's et al. study was not

Table $4 \mathrm{VOI}$ ratio in AF patients and CG (mean \pm SD)

\begin{tabular}{llll}
\hline VOI & $\begin{array}{l}\text { AF } \\
(n=224)\end{array}$ & $\begin{array}{l}\text { CG } \\
(n=40)\end{array}$ & $p$ \\
\hline RSPV & $0.93 \pm 0.2$ & $0.94 \pm 0.2$ & NS \\
RIPV & $0.91 \pm 0.1$ & $1.02 \pm 0.2$ & $<0.005$ \\
LSPV & $0.83 \pm 0.2$ & $0.81 \pm 0.2$ & NS \\
LIPV & $0.79 \pm 0.2$ & $0.75 \pm 0.2$ & NS \\
\hline
\end{tabular}

$R S P V$ right superior pulmonary vein, RIPV right inferior pulmonary vein, $L S P V$ left superior pulmonary vein, LIPV left inferior pulmonary vein conclusive - he presented only statistical trends of the relation between PV anatomy and PVI efficacy [10].

In contrast, a few studies state the opposite [25], eg. a recent paper by Heeger et al. analizes the outcome of PVI using cryoballoon in 74 pts. with left common ostia PV; he found the efficacy of PVI equal in the study and the control group, although in some cases the presence of left CO of PV required a different PVI technique [26].

The lack of decisive opinion on PV anatomy calls for carrying out more studies with larger groups. The full recognition of AF pathophysiology, LA and PV anatomy and remodelling are essential for optimalization of $\mathrm{AF}$ treatment strategies.

It should be noted that rare pathological findings were observed in pts. with AF initially qualified for PVI. The precise left atrium visualization helped us to properly qualify the patient for ablation and avoid procedural complications.

Anatomical anomalies of LA and PV may potentially increase the periprocedural complications and affect PVI efficacy [27]. We consider identifying anatomy anomalies as an important factor that may change the operator's approach and modify the therapeutic strategy.

The main limitation of this paper is the number of patients in the control group.

\section{Conclusions}

The anomalies of pulmonary vein anatomy occur more often in pts. with atrial fibrillation. They can be defined as an image biomarkers of atrial fibrillation. The right additional pulmonary vein was the most common anomaly detected in AF patients as well as enlarged diameters of the LA and PV ostia. The differences of pulmonary veins anatomy apply not only to the number of PVs but also to the localization and shape of PV's ostia.

\section{Abbreviations}

AF: Atrial fibrillation; APV: Additional pulmonary vein; CG: Control group; CMR: Cardiac magnetic resonanse; CO: Common ostia; EB: Early branching; ECG: Electrocardiogram; MSCT: Multi-slice computer tomography; PTS: Patients; PVI: Pulmonary vein isolation; SD: Standart deviation; VOI: Venous ostium index

Funding

The authors received no specific funding for this work.

\section{Availability of data and materials}

The datasets used and/or analysed during the current study are available from the corresponding author on reasonable request.

\section{Authors' contributions}

MS1 conceived the idea for the study, supervised it, analyzed the CT scans and wrote the manuscript with IWS. AH and SN helped with CT scans evaluation. TS created the data base, drafted the article and performed the statistical analysis. MS2, AMWW and KMS revised the article. All authors were involved in data collection. All authors edited and approved the final version of the manuscript. 


\section{Ethics approval and consent to participate}

The study was approved by the ethics committee (Medical University of Silesia ethics comittee) and conformed to the Declaration of Helsinki. An informed written consent was obtained from every patient enrolled in the study.

\section{Consent for publication}

n/a

\section{Competing interests}

The authors declare that they have no competing interests.

\section{Publisher's Note}

Springer Nature remains neutral with regard to jurisdictional claims in published maps and institutional affiliations.

\section{Author details}

${ }^{1}$ First Department of Cardiology, School of Medicine in Katowice, Medical University of Silesia, Katowice, Poland. ${ }^{2}$ Department of Cardiology, School of Health Sciences, Medical University of Silesia, Katowice, Poland. ${ }^{3}$ Unit of Noninvasive Cardiovascular Diagnostics, Medical University of Silesia, Katowice, Poland.

Received: 23 January 2018 Accepted: 5 July 2018

Published online: 13 July 2018

\section{References}

1. Calkins H, Kuck KH, Cappato R et al. 2012 HRS/EHRA/EACS expert consensus statement on catheter and surgical ablation of atrial fibrillation: recommendations for patient selection, procedural techniques, patients management and follow-up, definitions, endpoints and research trial design: a report of the Heart Rhythm Society (HRS) task force on catheter and surgical ablation of atrial fibrillation. Heart Rhythm; 2012 9: 632-696.

2. Kato $R$, Lickfett $L$, Meininger $G$, et al. Pulmonary vein anatomy in patients undergoing catheter ablation of atrial fibrillation. Circulation. 2003;107:2004-10.

3. Jongbloed M, Bax J, Lamb H, et al. Multislice computed tomography versus intracardiac echocardiography to evaluate the pulmonary veins before radiofrequency catheter ablation of atrial fibrillation. J Am Coll Cardiol. 2005:45:43-50

4. Tekbas G, Ekici F, Tekbas E, et al. Evaluation of pulmonary vein variations in the middle pulmonary lobe with 64-slice multidetector computed tomography. Eur Rev Med Pharmacol Sci. 2011;15(12):1395-400

5. Yokokawa $M$, Olgun $H$, Sundaram $B$, et al. Impact of preprocedural imaging on outcomes of catheter ablation in patients with atrial fibrillation. J Interv Card Electrophysiol. 2012;34(3):255-62.

6. Akiba T, Mirikawa T, Inagaki T, et al. A new classification for right top pulmonary vein. Ann Thorac Surg. 2013 Apr;95(4):12277-30.

7. Kubala M, Hermida JS, Nadij G, et al. Normal pulmonary veins anatomy is associated with better AF-free survival after cryoablation as compared to atypical anatomy with common left pulmonary vein. Pacing Clin Electrophysiol. 2011;34(7):837043.

8. Woźniak-Skowerska I, Skowerski M, Wnuk-Wojnar A, et al. Comparison of pulmonary veins anatomy in patients with and without atrial fibrillation: analysis by multislice tomography. Int J Cardiol. 2011;146(2):181-5.

9. Bittner A, Monnig G, Vagt A, et al. Pulmonary vein variants predispose to atrial fibrillation: a case-control study using multislice enhanced computed tomography. Europace. 2011;13:1394-400.

10. Mulder AAW, Wijffels MCEF, Wever EFD, et al. Pulmonary vein anatomy and long-term outcome after multi-electrode pulmonary vein isolation with phased radiofrequency energy for paroxysmal atrial fibrillation. Europace. 2011;13:1557-61.

11. Chen J, Yang Z, Xu H, et al. Assessments of pulmonary vein and left atrial anatomical variants in atrial fibrillation patients for catheter ablation with cardiac CT. Eur Radiol. 2017;27:660-70

12. Tekbas G, et al. Evaluation of pulmonary vein variations and anomalies with 64 slice multi detector computed tomography. Wien Klin Wochenschr. 2012;124:3-10

13. Baran J, Piotrowsk R, Sikorska A, et al. Impact of pulmonary vein ostia anatomy on efficacy of cryoballoon ablation for atrial fibrillation. Heart Beat Journal. 2016;1:65-70. https://doi.org/10.24255/hbj/68162.
14. Tekbas G, Gumus $\mathrm{H}$, Onder $\mathrm{H}$, et al. Evaluation of pulmonary vein variations and anomalies with 64 slice multi detector computed tomography. Wiener klinische Wochenscgrift. 2012;124:3-10.

15. Manghat $\mathrm{NE}$, Mathias $\mathrm{HC}$, Kakani N, et al. Pulmonary venous evaluation using electrocardiogram-gated 64-detector row cardiac CT. Br J Radiol. 2012;85(1015):965-71.

16. Schafer D, Meyer C, Bullens R, et al. Limited angle C-arm tomography and segmentation for quidance of atrial fibrillation ablation procedures. Med Imae Comput Assist Interv. 2012;15:634-41.

17. De Potter T, Bardhaj G, Viggiano A, et al. Rotational angiography as a Periprocedural imaging tool in atrial fibrillation ablation. Arrhythm Electrophysiol Rev. 2014;3(3):173-6

18. Bonso A, Fantinel M, Scalchi $G$, et al. Left atrial model reconstruction in atrial fibrillation ablation: reliability of new mapping and complex impedance systems. Europace. 2016;

19. Marom E, Herdon J, Kim YH, et al. Variations in pulmonary venous drainage to the left atrium implication for radiofrequency ablation. Radiology. 2004; 230:824-9.

20. Thai WE, Wai $B$, Lin $K$, et al. Pulmonary venous anatomy imaging with lowdose, prospectively ECG-triggered, high-pitch 128-slice dual-source computed tomography. Circ Arrhythm Electrophysiol. 2012;5(3):521-30.

21. Ratajczak P, Sławińska A, Martynowska-Rymer I, et al. Anatomical evaluation of the pulmonary veins and the left atrium using computed tomography before catheter ablation: reproducibility of measurements. Pol J Radiol. 2016;81:228-32.

22. Tsao HM, Wu MH, Yu WC, et al. Role of right middle pulmonary vein in patients with paroxysmal atrial fibrillation. J Cardiovasc Electrocardiol. 2001; 12:1353-7.

23. Streb W, Jarski P, Przybylski R, et al. Right atrial diverticulum in an adult person. Kardiol Pol. 2007;65:1090-3.

24. Sohns C, Sohns JM, Bergau $L$, et al. Pulmonary vein anatomy predicts freedom from atrial fibrillation using remote magnetic navigation for circumferential pulmonary vein ablation. Europace. 2013:15:1136-42.

25. Dennis W, Tops LF, Delgado $V$, et al. Effect of pulmonary vein anatomy and left atrial dimensions on outcome of circumferential radiofrequency catheter ablation for atrial fibrillation den Uijl. American Journal of Cardiology. 2011:107:243-9.

26. Heeger C, Tscholl V, Wissner E, et al. Acute efficacy, safety, and long-term clinical outcomes using the second-generation cryoballoon for pulmonary vein isolation in patients with a left common pulmonary vein: a multicenter study. Heart Rhythm. 2017;14:1111-8.

27. Sorgente A, Chierchia G, De Battista C, et al. Pulmonary vein ostium shape and orientation as possible predictors of occlusion in patients with drugrefractory paroxysmal atrial fibrillation undergoing cryoballoon ablation. Europace. 2011;13:205-12.

\section{Ready to submit your research? Choose BMC and benefit from:}

- fast, convenient online submission

- thorough peer review by experienced researchers in your field

- rapid publication on acceptance

- support for research data, including large and complex data types

- gold Open Access which fosters wider collaboration and increased citations

- maximum visibility for your research: over $100 \mathrm{M}$ website views per year

At BMC, research is always in progress.

Learn more biomedcentral.com/submissions 